\title{
Caracterización de Pacientes con Atresia Esofágica
}

\author{
Characterization of Patients with Esophageal Atresia \\ Luis Gerardo Padilla Meza* Juan Craniotis Ríos** Juan José Guerra***
}

\section{RESUMEN}

Antecedentes: La atresia de esófago (AE) es la anomalía congénita del esófago más importante, tiene una incidencia mundial de 1:3500 nacidos vivos. A nivel mundial la mortalidad es de $10 \%$ pero en países cercanos como Costa Rica es del 16\%. Objetivo: Identificar las características de los pacientes con AE atendidos en el Hospital Mario Catarino Rivas. Pacientes y métodos: Se realizó un estudio de tipo descriptivo, transversal en el Hospital Nacional Dr. Mario Catarino Rivas (HNMCR). Se obtuvo la información del expediente clínico. Se incluyeron en el estudio los 23 recién nacidos con diagnóstico de $A E$ que se atendieron en el área de Emergencia de Pediatría y sala de Cuidados Intensivos Neonatales del período de enero del 2013 a agosto del 2014. Resultados: El 78\% de los niños con AE fueron varones ( $n=18)$. El 70\% $(n=16)$ de las madres realizó adecuado control prenatal y el $35 \%(n=8)$ se realizó ultrasonido (USG). Se realizó el diagnóstico entre las 24-48 horas en el $57 \%$ de los pacientes $(n=13) .74 \%$ $(n=17)$ nacieron a término. El tipo de atresia más frecuente fue el tipo III en 15 niños (65\%). Fallecieron 19 de los niños (82\%). Conclusiones: En nuestro sistema de salud la tasa de supervivencia a una AE es baja, y el control prenatal no aporta relevancia para el diagnóstico de esta patología.

\section{PALABRAS CLAVE}

Atresia Esofágica, Fístula Esofágica, Mortalidad.

\section{ABSTRACT}

Background: the esophageal atresia is the

\footnotetext{
*Médico Residente de tercer año de Postgrado de Pediatría. Universidad Nacional Autónoma de Honduras en el Valle de Sula. UNAH-VS

${ }^{* *}$ Cirujano Pediatra, Hospital Nacional Mario Catarino Rivas. HNMCR

***Epidemiólogo, UNAH-VS

Dirigir correspondencia: lupahn@hotmail.com

Recibido: 13/Dic/2015 Aceptado:27/Jul/2015
}

most important congenital abnormality of the esophagus, with a worldwide incidence of 1 : 3500 live births. Worldwide mortality is $10 \%$ but in nearby countries like Costa Rica is $16 \%$. Objective: To identify the characteristics of patients with esophageal Atresia in the Hospital Mario Catarino Rivas. Patients and Methods: A descriptive, cross-sectional study was conducted at the Hospital Nacional Dr Mario Catarino Rivas (HNMCR), the information was obtained from the clinical records. 23 newborns were included in the study admitted at the Pediatric Emergency and Neonatal intensive care unit from January 2013 thru August 2014. Results: $78 \%$ of children with AE were male $(n=18) .70 \%$ $(n=16)$ of mothers made adequate prenatal care and $35 \%(n=8)$ ultrasound (USG) was performed. Diagnosis within 24-48 hours was performed in $57 \%$ of patients $(n=$ 13). $74 \%(n=17)$ were born at term. The most frequent was the type III atresia in 15 children (65\%). They killed 19 children (82\% Conclusion: In our health system the survival rate to an EA is low, and does not provide prenatal care relevant to the diagnosis of this pathology.

\section{KEYWORDS}

Esophageal Atresia, Esophageal Fistula, Mortality.

\section{INTRODUCCION}

Aunque la tasa de supervivencia total de niños con atresia de esófago a nivel mundial actualmente supera el $90 \%$, esta condición todavía continúa siendo responsable de una significativa mortalidad, principalmente como consecuencia de otras anomalías congénitas asociadas evidenciadas por múltiples estudios a nivel mundial; y esto resulta ser mayor en países en vías de desarrollo. ${ }^{(1)}$ 
La historia documentada de la atresia esofágica se inició en el año 1670 con la descripción publicada por William Durston. En1697 Thomas Gibson describió por primera vez la forma típica de la anomalía. ${ }^{(1-3)}$ Las variaciones anatómicas de esta patología fueron descritas por Vogt en 1929, y luego Ladd y Gross simplificaron su clasificación. ${ }^{(4-5)}$ La AE ocurre por alteración en la embriogénesis probablemente durante las primeras 6 semanas de vida fetal. ${ }^{(6)}$

Más del 50\% de los casos de atresia de esófago con fístula están asociados con otras anomalías, y hasta el $10 \%$ de los casos específicos se encuentran trastornos cromosómicos. La incidencia de atresia de esófago, con o sin fístula traqueo-esofágica, es 1:3.500 recién nacidos vivos. Se presentan casos en hermanos e hijos de padres con atresia esófago y con una mayor frecuencia en gemelos; sin embargo, aún no existe un patrón hereditario establecido. ${ }^{(7-8)} \mathrm{La}$ clasificación original de Vogt (1929), modificada por Ladd en 1944 y Gross en 1953 se sigue utilizando hasta nuestros días, donde la forma más común se presenta en $85 \%$ a $90 \%$ de los casos y consiste en atresia de esófago proximal con una fístula traqueo esofágica distal. La forma pura de atresia de esófago es la segunda en importancia y es vista en 5\% a 7\%. La fístula traqueo esofágica sin atresia de esófago (fístula en $\mathrm{H}$ ) se presenta sólo en un $2 \%$ a $6 \%$ de los casos. ${ }^{(9)}$

Las malformaciones congénitas coexisten en aproximadamente la mitad de los pacientes con atresia de esófago, siendo las anomalías cardíacas las más comunes, seguidas de las musculo esqueléticas, anales y genitourinarias. La presencia de malformaciones cardíacas es el mayor determinante de mortalidad. ${ }^{(10,11)}$

Actualmente el diagnóstico, en su mayoría, se realiza en forma prenatal al observarse en la ecografía una disminución o ausencia de la burbuja gástrica asociada a polihidramnios, (12-13) clínicamente se observa un recién nacido con salivación excesiva y dificultad respiratoria sin poder introducirle una sonda nasogástrica.
La evaluación se complementa con radiografía toracoabdominal para definir la presencia de fístula traqueo-esofágica. El diagnóstico precoz permite un tratamiento oportuno, con lo cual se logra disminuir las complicaciones y se reduce la morbimortalidad. ${ }^{(14-15)}$ El tratamiento inmediato incluye medidas para prevenir la aspiración y desarrollo de neumonitis. ${ }^{(16)}$

La atresia de esófago no es una emergencia quirúrgica, por lo que el recien nacido se llevará a cirugía una vez que se encuentre estable, se determine el tipo y la gravedad de las malformaciones asociadas y se establezca un plan de tratamiento quirúrgico. ${ }^{(14)}$

La supervivencia por lo general es buena, en la actualidad es superior a $90 \%,{ }^{(17)}$ y aun asi hay importante mortalidad en los paises en desarrollo por la falta de insumos y por no contar con adecuadas salas de cuidados intensivos neonatales.

En Honduras contamos con 4 estudios sobre las atresias de esófago dirigidos al manejo y técnicas quirúrgicas, realizados en la ciudad capital en el Hospital Escuela Universitario, (4-5,18-19) no existe otro estudio que exponga la realidad de lo que sucede en nuestros hospitales. Es por eso que se decide realizar este estudio en el Hospital Nacional Dr. Mario Catarino Rivas, con el objetivo de caracterizar a los pacientes con atresia de esófago, ya que no se cuenta con la información para hacer una comparación a las estadisticas que se muestran a nivel mundial con respecto a nuestra realidad hospitalaria.

\section{PACIENTES Y MÉTODOS}

Se realizó un estudio transversal, de tipo descriptivo, acerca de la caracterización de pacientes con AE atendidos en el HNMCR en el periodo enero 2013 - agosto 2104.

Se incluyó a todos los neonatos con diagnóstico de $A E$, atendidos en sala de Emergencia Pediátrica y la Unidad de Cuidados Intensivos Neonatal del HNMCR. 
Se incluyeron 23 casos con diagnóstico de AE. El instrumento utilizado para la recolección de datos fue el cuestionario, elaborado con preguntas abiertas y cerradas que incluían: edad de la madre, si se realizó USG, cuantos USG se realizó, si se hizo diagnóstico de atresia de esófago y la edad gestacional al momento del diagnóstico ultrasonográfico, edad gestacional al momento del nacimiento, edad al momento del diagnóstico, tipo de atresia encontrada, si se realizó cirugía y cuál fue el tipo de cirugía realizada, edad al momento de la cirugía, malformaciones congénitas asociadas, condición de egreso, si falleció cual fue la causa de muerte, días de estancia intrahospitalaria. Se realizó una prueba piloto para validar dicho cuestionario; se obtuvo la información del expediente clínico de cada paciente. Una vez recolectada la información, el procesamiento de datos se realizó a través del programa Excel 2012 con la obtención de frecuencias simples, porcentajes y proporciones que dieron salida a los objetivos del estudio.

\section{RESULTADOS}

En cuanto al control prenatal que recibieron las madres; $70 \%(n=16)$ tuvieron más de 4 controles, $17 \%(n=4)$ menos de 4 y $13 \%(n=3)$ no tuvo control prenatal. Solo $35 \%$ (8) se realizaron al menos un USG, ninguno de éstas tuvo sospecha diagnóstica de atresia de esófago.

De los 23 recién nacidos, $74 \%(n=17)$ eran de término y $26 \%(n=6)$ pretérmino. La distribución por género fue de $78 \%(n=18)$ del sexo masculino y de $22 \%(n=5)$ femenino, lo que nos da una relación de 3.5:1.

La edad al momento del diagnóstico en $57 \%$ $(n=13)$ pacientes fue en un rango de 24- 48 horas, 30\% $(n=7)$ menos de 24 horas de vida, $4 \%(n=1)$ entre $72-96$ horas y $9 \%(n=2)$ más de 5 días de vida al momento del diagnóstico. De los pacientes a los que se les detectó la $A E$ en las primeras 24 horas 5 nacieron en el HNMCR, los 18 niños restantes fueron referidos de otros centros hospitalarios. El tipo de atresia que más se encontró fue la tipo III. Ver gráfico No 1.

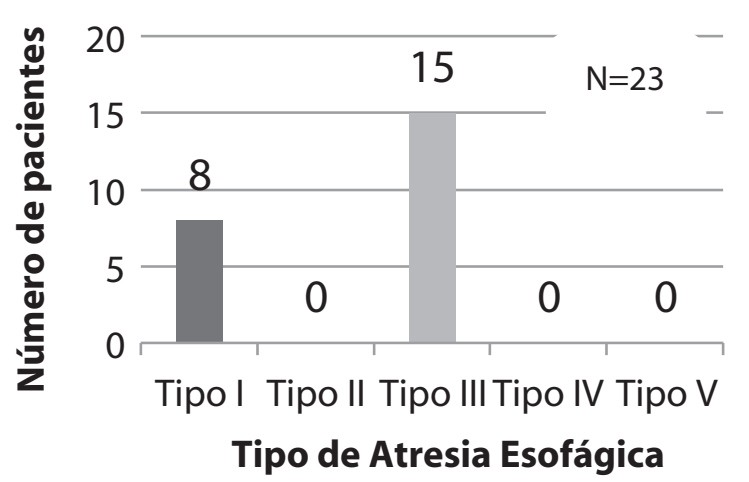

Gráfica No1. Tipos de Atresia de esófago encontradas

Fuente: Investigación atresia de esófago.

Se realizó cirugía a 21 pacientes. Se realizó la cirugía definitiva en $52.3 \%(n=11)$ y la esofagostomia mas gastrostomia con ligadura de fistula en un $47.7 \%(n=10)$, los otros 2 pacientes fallecieron antes de brindarle un tratamiento quirúrgico.

Un $76 \%(n=16)$ tuvo una espera de $24-48$ horas para ser operado y un $10 \%(n=2)$ esperó entre 48-72 horas para la cirugía y $14 \%(n=3)$ esperaron menos de 24 horas para ser operados. Al $82.6 \%(n=19)$ no se les investigó por malformaciones asociadas debido a que el hospital no cuenta con las herramientas diagnósticas adecuadas, sin embargo se encontraron 4 malformaciones: una con sospecha de cardiopatia, una malfomación anorectal tipo ano imperforado, un sindrome de Down y otro niño con orejas de implantacion baja, micropene y criptorquidea bilateral.

La mortalidad en el estudio fue de $82 \%(n=18)$. La principal causa de muerte fue la sepsis en el $89 \%(n=16)$ de los casos y de estas la causa más importante fue la neumonía en 39\% $(n=7)$. El tiempo de estancia intrahospitalaria fue de 6-10 días en un 39\% $(n=9)$ de los casos, el 9\% $(n=2)$ estuvieron mas de 20 días hospitalizados. Ver Tabla No.1 
Tabla No. 1. Días intrahospitalarios de los pacientes con atresia de esófago.

\begin{tabular}{lcc}
\hline \multicolumn{1}{c}{ Estadía } & $\mathbf{n}$ & Porcentaje \\
\hline 1-5 días & 5 & $22 \%$ \\
6- 10 días & 9 & $39 \%$ \\
11-15 días & 7 & $30 \%$ \\
16-20 días & 0 & $0 \%$ \\
>20 días & 2 & $9 \%$ \\
Total & 23 & $100 \%$ \\
\hline
\end{tabular}

Fuente: Investigación atresia de esófago.

\section{DISCUSIÓN}

La atresia de esófago es talvez una de las patologías quirúrgica clásicas de la cirugía pediátrica que ha tenido un desarrollo muy importante en los últimos 20 años en relación con la sobrevida. Analizaremos en primer lugar las características encontradas en la población en estudio, la edad gestacional al nacer, los recien nacidos fueron de termino por clínica en $76 \%$ de los casos pero también se presentó en recién nacidos pretérmino en $26 \%$, esto último coincide con la literatura internacional que hace referencia a $25-30 \%$ se presenta en prematuros. ${ }^{(7)}$

Solo un $70 \%$ tuvo control prenatal adecuado; teniendo en cuenta que mas de 4 controles es considerado como un exitoso control prenatal; de estos solo $40 \%$ se realizó USG prenatal, y unicamente un paciente tuvo el antecedente de polihidramnios, ninguno tuvo la sospecha diagnostica radiológica. La literatura refiere que la detección prenatal de AE por ecografía depende de la pericia del ultrasonografista y se basa en el hallazgo de una burbuja de estómago pequeño o ausente asociado a polihidramnios materno, pero esta asociación solo tiene un valor predictivo del $40 \% .{ }^{(7,8)}$ En Costa Rica se evidenció que de 59 pacientes estudiados un $6.1 \%$ tuvo la sospecha diagnóstica radiologica de atresia de esófago. ${ }^{(13)}$ Como se puede apreciar el control prenatal, tanto de forma clínica como radiológica, no aporta relevancia para el diagnóstico de esta patología, pero si resalta el hecho que no se debe perder la oportunidad de diagnosticar esta entidad de forma prenatal, ya que mejora las posibilidades de referir a la paciente a centros de atención de mayor complejidad, por lo que se debe insistir en realizar el ultrasonido durante el embarazo. La AE predominó en los recien nacidos masculinos con una relación hombre mujer de 3.5:1, a diferencia de lo descrito en la literatura que hace referencia a un ligero predominio masculino. ${ }^{(1,7)}$

La literatura internacional refiere que el diagnóstico debe ser inmediato al nacer pero hay estudios, por ejemplo, de países como Chile, México, Costa Rica, que muestran que el diagnóstico tuvo un promedio de $1-2$ días. ${ }^{(7,13,20)}$ En los niños hospitalizados en el HNMCR, el diagnóstico de $A E$ se realizó entre las 24-48 horas de vida en $57 \%$ de los pacientes, y el $9 \%$ el diagnóstico se realizó después de los 5 días de vida. Para minimizar fallos en el diagnóstico, se debe verificar la permeabilidad del esófago en todos los niños al momento de nacer.

El tipo de atresia que predominó fue la tipo III (65\%) y seguido de la tipo I (35\%) estos resultados no muestran diferencia con la literatura internacional en cuanto a la distribución de frecuencia, pero si se muestra diferencia en los porcentajes ya que la tipo I es de $5-8 \%$ y la tipo III de $80-85 \% .^{(3)}$ Al $91 \%$ de los pacientes se le realizo cirugía, los demás fallecieron antes de practicarle la atención quirúrgica. No hay una diferencia marcada con respecto a los tipos de cirugía realizados en el departamento de cirugía pediátrica de nuestro hospital, se practicó a 10 de los pacientes que tenían AE tipo III la cirugía definitiva y la otra mitad de los pacientes ( 3 pacientes tipo III y 8 pacientes con $\mathrm{AE}$ tipo I) se le realizó esofagostomía mas gastrostomía y ligadura de fistula. En el caso de la $\mathrm{AE}$ tipo I, se puede realizar la corrección directa con la trasposición de colon, que se está realizando con éxito en el Salvador, México y países del primer mundo, pero aun no estamos en condiciones para realizar estos procedimientos en nuestro hospital. En $82.6 \%$ pacientes no se investigó malformaciones asociadas por falta de instrumentos diagnósticos en el hospital, el paciente con cardiopatía no se realizó ecocardiograma, siendo su diagnóstico meramente clínico, por lo que resultaría difícil tratar de buscar asociación entre las malformaciones y el 
pronóstico de los pacientes. El tiempo transcurrido entre el diagnóstico y la cirugía fue de 24-48 hrs en $76 \%(16)$ y $10 \%(2)$ entre $48-72$ hrs. Resultados que no concuerdan con otros estudios realizados en Chile por ejemplo que hace referencia a que $60 \%$ fue operado antes de las 24 horas del diagnóstico. ${ }^{(20)}$

Internacionalmente la sobrevida para esta patología es de alrededor del $90 \%{ }^{(3,7)}$ pero en nuestro estudio se encontró una sobrevida únicamente del $18 \%$, esto se debe a la alta incidencia de infecciones nosocomiales, siendo la sepsis la causa de mortalidad de los pacientes, y complicaciones propias de la AE como ser la neumonía. En su totalidad los recién nacidos fueron manejados en la unidad de cuidados intensivos neonatales, de los 4 pacientes que sobrevivieron, 1 fue manejado en la UCIP con una estancia hospitalaria de 29 días. Otra sobre viviente fue una paciente que luego de ser operada se trasladó al Instituto Hondureño de Seguridad Social.
En conclusión; este estudio evidencia que no se está cumpliendo a cabalidad la atención inmediata del recién nacido en los centros que refieren pacientes a nuestro hospital ya que el diagnóstico de la enfermedad se da después de las 24 horas de nacido, muestra de eso es que se reciben pacientes hasta con 5 días de vida al momento del diagnóstico. En el estudio no se puede caracterizar las malformaciones congénitas asociadas debido a la falta de herramientas diagnósticos en el hospital. Aunque la sobrevivencia de esta patología es muy alta internacionalmente incluso en países cercanos al nuestro como Costa Rica; nuestro hospital tiene una mortalidad tan alta como la que se presentaba en los años 50 . Se recomienda que a partir de este estudio se realice un protocolo para la atención del recién nacido con atresia de esófago para disminuir la mortalidad en el hospital y posteriormente dar seguimiento con nuevos estudios para poder comprender y obtener más datos de estos pacientes.

\section{BIBLIOGRAFÍA}

1. Reyes RR, Muñiz EJ, Polo Al, Alvaredo SMA, Pascual HAM, Hernández FNM. Relación entre el peso al nacer y la mortalidad por atresia esofágica. Rev Cubana Pediatr. 2014 [citado 02 noviembre 2014]; 86(2):

Disponible en: http://scielo.sld.cu/scielo. php? script $=$ sci_arttext $\&$ pid $=$ S0034753 $12014000200004 \&$ Ing=es.

2. Depalma D. Atresia de esófago (primeros antecedentes). Rev Hosp Niños B Aires Diciembre 2012 [citado 02 noviembre 2014]; 54,(247): 249-250 disponible en: http://revistapediatria.com.ar/wp-content/ uploads/2013/01/249-250Artresia.pdf.

3. Howard C. Filston, Nicholas A. Shorter. Atresia Esofágica y Malformaciones Traqueoesofágica. En: Ashcraft. Murphy. Sharp. Sigalet. Snyder. Cirugía Pediatrica. 3ra edición. McGraw Hill. 2001. 370-392.

4. Lizardo J.R. Atresia de Esófago Presentación de 5 casos y Revisión de la Literatura. Rev. méd. Hondur. 1993; 61(2):71-4.

5. Lovo S. Atresia de esófago con fístula traqueo-esofágica primer caso con sobrevida en Honduras. Honduras Pediátrica. 1969; 4(2):114-119.

6. loannides AS, Copp AJ. Embryology of esophageal atresia. Seminars in Pediatric Surgery. [Revista en internet] 2009 [citado 02 mayo 2013]; 18(1):2-11. doi: 10. 1053/ j.sempedsurg.2008.10.002 Disponible en: http://www.ncbi.nlm.nih.gov/pmc/ articles/PMC3641483/.

7. García Heladia, Franco Gutiérrez Mario. Manejo multidisciplinario de los pacientes con atresia de esófago. Bol. Med. Hosp. Infant. Mex. [revista en la Internet]. 2011 Dic [citado 2015 Jun 30]; 68(6): 467-475. Disponible en: http://www.scielo.org.mx/ scielo.php?script=sci_arttext\&pid=S1665$11462011000600010 \&$ Ing $=$ es. 
8. Pinheiro PFM, Simões e Silva AC, Pereira RM. Current knowledge on esophageal atresia. World Journal of Gastroenterology: WJG. [Revista en internet] 2012 [citado 06 junio 2013];18(28), 3662-3672. doi:10.3748/wjg. v18. i28.3662 http://www.ncbi.nlm.nih.gov /pmc/articles/PMC3406418/.

9. Martínez-Ferro M. Atresia de Esófago "Long Gap": Definición y Conducta Quirúrgica Moderna. Rev. Med. Clin. CONDES - 2009[citado 13 noviembre 2013]; 20(6) 797 - 803. Disponible en: http://www.clinicalascon des.com/area-academica/pdf/MED_20_ 6/009_atresia_esofago.pdf.

10. Contador M. Correia G. Recién Nacido Quirúrgico.En:Rostion C.G.Cirugía Pediátrica. Santiago de Chile: Mediterraneo; 2001. 284-299.

11. Knottenbelt G, Skinner A, Seefelder C. Tracheo-oesophageal fistula (TOF) and esophageal atresia (OA). Best Practice \& Research Clinical Anesthesiology. 2010 [citado 4 enero 2014]; 24(3):387-401. Disponible en: http://www.sciencedirect.com/ science/article/pii/S1521689610000273.

12. Reyes Rodríguez Roberto, Muñiz Escarpanter José, Polo Amorín Ismael, Alvaredo Soria Manuel Alejandro, Armenteros García Abel, Hernández Fernández Neisy María. Anomalías congénitas asociadas a la atresia esofágica. Rev Cubana Pediatr [revista en la Internet]. 2014 [citado 2015 Jun 30] ; 86(1):6876. Disponible en: http://scielo.sld.cu/ scielo.php?script=sci_arttext $\&$ pid=S0034-753 $12014000100008 \&$ Ing=es.

13. Rodríguez PJA, Lazo BJ, Rivera CM. Características clínicas de los pacientes con diagnóstico de atresia de esófago. Acta pediátr costarric. 2010[citado 02 marzo 2014]; 22(2):86-94. Disponible en: http://www.bi nasss.sa.cr/revistas/apc/v22n2/art4.pdf.

14. Del Rio.GS, García GR. Neonato Quirúrgico.
En:Del Rio.GS, García G.R. Manual Básico de Cirugía Pediátrica. México. 2005. 114-120.

15. Holland AJ, Fitzgerald DA. Esophageal atresia and tracheo-oesophageal fistula: current management strategies and complications. Paediatric Respiratory Reviews. [Revista en internet] 2010[citado 03 noviembre 2014]; (11):100-107. doi: 10.1016/ j.prrv.2010.01.007. Disponible en: http:// www.ncbi.nlm.nih.gov/pubmed/20416546.

16. Montalvo MA, Porras RG. Atresia Esofágica y Fistula Traqueo esofágica. En: Montalvo MA, Álvarez SR. Guía de procedimientos de cirugía pediátrica. 2da edición. México. McGraw-Hill, 1999.53-63.

17. Koivusalo Al, Pakarinen MP, Rintala RJ. Modern outcomes of esophageal atresia: Single center experience over the last twenty years. J Pediatr Surg. [Revista en internet] 2013[citado 13 noviembre 2014]; 48(2): 297-303. doi: 10.1016/j.jpedsurg. 2012.11.007. http://www.ncbi.nlm.nih.gov/ pubmed/23414855

18. Lizardo BJR, Godoy MJG, Zavala ACR, Munguia LE, Ramos Al. Interposición de colon para atresia esofágica pura. Reporte de un caso y revisión de la literatura. Rev Med Hondur. 2005;73(2):72-76

19. Lizardo BJR, Godoy MJG, Figueroa $\mathrm{CH}$, Ramos Al. Fístula traqueoesofágica en $\mathrm{H}$. Reporte de un caso y revisión de la literatura; Rev Med Hondur 2006; 74(1): 31-34.

20. Fierro AC, Caro DM, Anzieta VJ, Butte BJM, González FP, Apablaza CJP. Atresia esofágica: Manejo quirúrgico en el Hospital Clínico Regional de Valdivia. Cuad. cir. (Valdivia). [Revista en internet] 2002[citado 04 Noviembre 2014];16 (1):20-25. DOI:10. 4206/ cuad.cir.2002.v16n1-04 Disponible en: http: //mingaonline.uach.cl/scielo.php?pid= S0718- 28642002000100004\&script=sci_ar ttext. 